Interventions (or Assessment of Risk Factors) In total 47 matches by basketball team A ( 9 players) and 41 matches by team B (7 players) were performed throughout the season. All training sessions and matches were executed as prescribed by the training and coaching staff without interference or manipulation.

Main Outcome Measurements The Oslo Sports Trauma Research Center (OSTRC) Questionnaire on Health Problems was used to collect data on injuries and illnesses on a weekly base. Furthermore, players filled in s-RPE and duration for each training and match. Prevalence's, severity scores, timeloss and total weekly load were compared for 1-match weeks and $\geq 2$-match weeks. The data were analyzed using multilevel modeling.

Results Prevalence of injuries and illnesses were $18.1 \%$ and 4.6\% for 1-match weeks and $17.2 \%$ and $3.3 \%$ for $\geq 2$-match weeks. Severity scores and time-loss were not significantly different for 1-match weeks compared to $\geq 2$-match weeks. Total weekly load was lower during $\geq 2$-match weeks compared to 1-match weeks.

Conclusions No significant differences for injuries and illnesses were observed between 1 -match weeks and $\geq 2$-match weeks. Coaches appeared to reduce training load to compensate for multiple matches during short-term match congestion.

\section{EVALUATION OF IN-EAR SENSOR SYSTEMS FOR QUANTIFYING HEAD IMPACTS IN YOUTH FOOTBALL}

\begin{abstract}
${ }^{1,2}$ Stian Bahr Sandmo, ${ }^{3,4,5}$ Andrew S Mclntosh, ${ }^{1}$ Thor Einar Andersen, ${ }^{6,7}$ Inga K Koerte, ${ }^{1}$ Roald Bahr. 'Oslo Sports Trauma Research Center, Norwegian School of Sport Sciences, Oslo, Norway; ${ }^{2}$ Faculty of Medicine, University of Oslo, Oslo, Norway; ${ }^{3}$ School of Engineering and ACRISP, Edith Cowan University, Joondalup, Australia; ${ }^{4}$ Monash University of Accident Research Centre, Monash University, Melbourne, Australia; ${ }^{5}$ Mclntosh Consultancy and Research, Sydney, Australia; ${ }^{6}$ Department of Child and Adolescent Psychiatry, Psychosomatic, and Psychotherapy, Ludwig-Maximilian-University, Munich, Germany; 'Department of Psychiatry, Psychiatry Neuroimaging Laboratory, Brigham and Women's Hospital, Harvard Medical School, Massachusets, USA
\end{abstract}

\subsection{6/bjsports-2021-IOC.11}

Background Wearable sensor systems have the potential to quantify head kinematic responses of head impacts in football. However, on-field use of sensors (e.g. accelerometers) remains challenging due to factors such as poor coupling to the head.

Objective To test the validity of a novel in-ear sensor for quantifying head-impact exposure in youth football.

Design Descriptive laboratory study/validation study.

Setting Youth football.

Participants Six male youth football players (15.3 \pm 0.3 years). Evaluations In step 1, the sensor was mounted to a Hybrid III headform (HIII) and impacted with a linear impactor or football (range: 9-144g). Accelerative forces, including peak linear acceleration (PLA), were obtained from both systems. In step 2 , six youth soccer players wore sensors during a structured training protocol including heading and non-heading exercises; in step 3, they completed two regular football sessions. For each recorded accelerative event, PLA outputs were compared to video.

Main Outcome Measurements In step 1, random and systematic error were calculated using HIII as reference. In steps 2 and 3 , mean values $( \pm S D)$ were calculated for $(1)$ all heading and (2) all non-heading events. Receiver operating characteristic curves were used to determine the sensor's discriminatory capacity in both on-field settings, and cut-off values for predicting outcomes were identified.

Results In step 1, random and systematic error were both $11 \%$ for PLA. In step 2, heading events resulted in higher absolute values (PLA $=15.6 \pm 11.8 \mathrm{~g})$ than non-heading events $(\mathrm{PLA}=4.6 \pm 1.2 \mathrm{~g})$; area under the curve (AUC) was 0.98. In step 3, AUC was $>0.99$. A 9g cut-off value yielded a positive predictive value of $100 \%$ in the structured training protocol vs. $65 \%$ in regular football sessions.

Conclusions The in-ear sensor displayed considerable random error and overestimated head impact exposures substantially. It showed excellent on-field accuracy for discriminating headings from other accelerative events, but secondary means of verifying events are still necessary.

\section{HEAD IMPACT EXPOSURE IN YOUTH FOOTBALL - ARE CURRENT INTERVENTIONS HITTING THE TARGET?}

${ }^{1,2}$ Stian Bahr Sandmo, ${ }^{1}$ Thor Einar Andersen, 3,4 Inga K Koerte, ${ }^{1}$ Roald Bahr. 'Oslo Sports Trauma Research Center, Norwegian School of Sport Sciences, Oslo, Norway; ${ }^{2}$ Faculty of Medicine, University of Oslo, Oslo, Norway; ${ }^{3}$ Department of Child and Adolescent Psychiatry, Psychosomatic, and Psychotherapy, Ludwig-Maximilian-University, Munich, Germany; ${ }^{4}$ Department of Psychiatry, Psychiatry Neuroimaging Laboratory, Brigham and Women's Hospital, Harvard Medical School, Massachusets, USA

\subsection{6/bjsports-2021-IOC.12}

Background Restrictions on heading in youth football have been implemented in the U.S. to limit head-impact exposure up until the age of 13 . However, current interventions remain poorly guided by evidence, and providing more accurate data on heading exposure is key to assess risk.

Objective To quantify heading exposure in youth football, assessing the effects of sex and age.

Design Prospective cohort study, based on direct observation of a convenience sample of football matches played during an international youth football tournament. The tournament was played without heading restrictions, with separate sex and age groups.

Setting Youth football.

Participants Male and female teams with players aged 11-19 years. A total of 267 matches was observed.

Independent Variables Sex and age. The elite senior level was included for comparison, using video analysis.

Main Outcome Measurements All heading events were registered, classified and assigned to individual players. Heading rates were calculated for each sex and age group.

Results We observed a total of 4011 player hours (1927 player hours for females, 2083 player hours for males). Males headed more frequently than females (2.7 vs. 1.8 headers/ player hour; $\mathrm{p}<0.001)$. Heading rates increased with age (ANOVA, p<0.001), approaching the elite senior level for players 16 years and older. There was substantial variation within teams for all age and sex groups, with the widest range (1-18 headers) observed for girls aged 19. Girls younger than 12 years had the lowest exposure, with an average of less than two players per team heading the ball, each with 1-2 headers.

Conclusions Age and sex influence head-impact exposure in youth football, and warrants careful consideration when 
introducing injury prevention measures. Males are more frequently exposed than females, heading rates increase with age, and there is substantial variation between players. Heading is a rare event in the youngest age groups, especially among females.

\section{HEAD IMPACT DOSES AND 'NO-GO' DEFICITS IN OLYMPIC AND NON-OLYMPIC SPORT ATHLETES}

${ }^{1}$ Adam Bartsch, ${ }^{2}$ Lori Glover, ${ }^{3}$ Jay Alberts, ${ }^{3}$ Jason Cruickshank, ${ }^{3}$ Elizabeth Jansen, ${ }^{3}$ Edward Benzel, ${ }^{3}$ Sergey Samorezov, ${ }^{4}$ Vincent Miele, ${ }^{5}$ Julian Bailes, ${ }^{6}$ Gerald McGinty, ${ }^{9}$ Steven Rowson, ${ }^{6}$ Christopher D'Lauro, ${ }^{8}$ Tyler Rooks, ${ }^{7}$ Kenneth Cameron, ${ }^{7}$ Megan Houston, ${ }^{9}$ Emily Kieffer, ${ }^{10}$ Laurel $\mathrm{Ng},{ }^{10}$ Kiran Mathews, ${ }^{14}$ Mikael Swaren, ${ }^{11}$ Norman Link, ${ }^{15}$ Missy Fraser, ${ }^{12}$ Jason P Mihalik, ${ }^{12}$ Johna Mihalik-Register, ${ }^{13} \mathrm{JT}$ Eckner, ${ }^{13}$ Annalise Lane. ${ }^{1}$ Prevent Biometrics, Minneapolis, USA; ${ }^{2}$ Fairview Sports Medicine, Minneapolis, USA; ${ }^{3}$ Cleveland Clinic, Cleveland, USA; ${ }^{4}$ University of Pittsburgh Medical Center, Pittsburgh, USA; ${ }^{5}$ NorthShore University Health System, Chicago, USA; ${ }^{6}$ USA Air Force Academy, Colorado Springs, USA; ${ }^{7}$ USA Military Academy, West Point, USA; ${ }^{8}$ USA Army Aeromedical Research Laboratory, Fort Rucker, USA; ${ }^{9}$ Virginia Polytechnic University, Blacksburg, USA; ${ }^{10}$ L3 Harris, San Diego, USA; ${ }^{11}$ University of California-Berkeley, Berkeley, USA; ${ }^{12}$ University of North Carolina, Chapel Hill, USA; ${ }^{13}$ University of Michigan, Ann Arbor, USA; ${ }^{14}$ Swedish Olympic Academy, Stockholm, Sweden; ${ }^{15}$ Texas State University, Frisco, USA

\subsection{6/bjsports-2021-IOC.13}

Background The relationship between head impact dose and observable functional deficits remains unclear. While studies have almost exclusively examined American football athletes, in Olympic athletes there are almost no data that explore this relationship.

Objective We aimed to use an impact monitoring mouthguard (IMM) to quantify head impact doses in Olympic and nonOlympic Sports, identifying high-energy impacts on video as 'No-go' per the NFL protocol.

Design Retrospective meta-analysis from American football, basketball, boxing, ice hockey, karate, lacrosse, mixed martial arts, rugby, tae-kwon-do, soccer.

Setting Sporting field

Patients (or Participants) 4500 impacts over 800 player-games.

Interventions (or Assessment of Risk Factors) Impact doses where the athlete was observed as 'no-go'.

Main Outcome Measurements Kinetic energy transfer (KE), risk-weighted exposure (RWE), peak scalar linear acceleration (PLA), peak scalar linear velocity (PLV), peak scalar angular acceleration (PAA), peak scalar angular velocity (PAV), impact location, impact direction, 'No-go' status.

Results The median KE, RWE, PLA, PAA, PLV and PAV was 5 $\mathrm{J}, 0.0002,20 \mathrm{~g}, 1500 \mathrm{rad} / \mathrm{s}^{2}, 10 \mathrm{rad} / \mathrm{s}$ and $1.5 \mathrm{~m} / \mathrm{s}$, respectively. American football athletes sustained the highest energy impact doses, boxers and mixed-martial artists sustained the highest cumulative dose for a day of competition. Ice hockey had the highest rate of 'no-go' impacts versus total impacts collected. Karate had the highest rotational kinematics. Of the nine (9) highest energy impacts to the side and rear of the head, all were 'no-go' impacts. Of the top eight (8) highest energy impacts to the front of the head, none were 'no-go' impacts.

Conclusions 'No-go' observations occurred in high energy impact doses to the rear and the sides of the head, while similar impact doses to the forehead seemed tolerable. Prospective Olympic athlete impact monitoring could help identify risky exposures.

\section{BIOMARKERS IN SERUM AFTER HEAD IMPACT EXPOSURE IN FOOTBALL}

${ }^{1,2}$ Stian Bahr Sandmo, ${ }^{3}$ Peter Filipcik, ${ }^{3}$ Martin Cente, ${ }^{3}$ Jozef Hanes, ${ }^{1}$ Thor Einar Andersen,

1,4,5Truls M Straume-Næsheim, ${ }^{1}$ Roald Bahr. 'Oslo Sports Trauma Research Center, Norwegian School of Sport Sciences, Oslo, Norway; ${ }^{2}$ Faculty of Medicine, University of Oslo, Oslo, Norway; ${ }^{3}$ Institute of Neuroimmunology, Slovak Academy of Sciences, Bratislava, Norway; ${ }^{4}$ Department of Orthopedic Surgery, Akershus University Hospital, Lørenskog, Norway; ${ }^{5}$ Department of Orthopedic Surgery, Haugesund Rheumatism Hospital, Haugesund, Norway

\subsection{6/bjsports-2021-IOC. 14}

Background The link between head-impact exposure in football and neurological sequelae remains controversial. Bloodbased biomarkers can provide valuable information in traumatic brain injuries, reflecting e.g. axonal damage.

Objective To investigate if repetitive headers or accidental head impacts in football could cause structural damage to the brain, detected as an increase in serum concentrations of neurofilament light $(\mathrm{NfL})$ or tau proteins.

Design Prospective cohort study.

Setting Elite division football.

Participants Male football players in the Norwegian premier league, including a total of 621 player seasons.

Independent Variables Short- and long-term exposures in football, with and without head impacts.

Main Outcome Measurements Baseline NfL and tau were measured in Norwegian professional football players in preseason. Then, the effect of short-term exposures (independent variable) was assessed by measuring biomarker levels (dependent variable) after three conditions: (1) high-intensity exercise, (2) repetitive headers, and (3) head-impact incidents in a match. The effect of long-term head impact exposure was assessed by comparing two groups with relative differences in previous concussions and headers at baseline (low vs. high levels).

Results We analyzed 354 samples. Mean $( \pm$ SD) NfL concentration was $6.8 \pm 2.6 \mathrm{pg} / \mathrm{mL}$; mean tau concentration was $1.2 \pm 0.7 \mathrm{pg} / \mathrm{mL}$. We observed no short-term effects on NfL after exposure from either of the three conditions. Tau levels rose in response to high-intensity exercise and repetitive headers, but not after accidental head-impact incidents; the highest absolute values were seen $1 \mathrm{~h}$ after high-intensity exercise. We did not detect any long-term effects on serum $\mathrm{NfL}$ or tau concentrations from previous concussions and headers.

Conclusions NfL and tau in serum were unaffected by head impacts in football, after both short-term and long-term exposure. Importantly, tau levels seem to rise in response to exercise, emphasizing the need for appropriate control groups in future studies. Our findings highlight important characteristics and limitations for using $\mathrm{NfL}$ and tau as biomarkers in sports.

\section{TACKLE CHARACTERISTICS ASSOCIATED WITH CONCUSSION IN BRITISH UNIVERSITY LEVEL RUGBY UNION}

${ }^{1}$ Simon Roberts, ${ }^{2}$ Simon Kemp, 'Luke Morgan, ${ }^{1,2}$ Keith Stokes. ${ }^{1}$ University of Bath, Bath, UK: ${ }^{2}$ Rugby Football Union, London, UK

10.1136/bjsports-2021-IOC.15 